\title{
A pilot study to assess the morphology and progression of
}

\section{non-carious cervical lesions}

Short title: the morphology and progression of non-carious cervical lesions

Iori Sugita ${ }^{a}$, Syozi Nakashima ${ }^{\mathrm{a}}$, Asaomi Ikeda ${ }^{\mathrm{b}}$, Michael F. Burrow ${ }^{\mathrm{c}}$, Toru Nikaido ${ }^{\mathrm{a}}$, Shisei Kubo $^{\mathrm{d}},{ }^{*}$, Junji Tagami $^{\mathrm{a}}$, Yasunori Sumi ${ }^{\mathrm{e}}$

${ }^{a}$ Department of Cariology and Operative Dentistry, Graduate School of Medical and Dental Sciences, Tokyo Medical and Dental University, 1-5-45 Yushima, Bunkyo-ku, Tokyo 113-8510, Japan

${ }^{\mathrm{b}}$ Clinical Oral Science, Department of Oral Health Care Sciences, Faculty of Dentistry, Tokyo

Medical and Dental University, 1-5-45 Yushima, Bunkyo-ku, Tokyo 113-0034, Japan

${ }^{\mathrm{c}}$ Department of Biomaterials, Melbourne Dental School, University of Melbourne, 720 Swanston St, Victoria, 3010, Australia

${ }^{\mathrm{d}}$ Medical Education Development Center, Nagasaki University Hospital , Nagasaki, 1-7-1

Sakamoto, Nagasaki 852-8588, Japan

${ }^{\mathrm{e}}$ Division of Oral and Dental Surgery, Department of Advanced Medicine, National Hospital for Geriatric Medicine, National Center for Geriatrics and Gerontology, 7-430 Morioka, Obu, Aichi 474-8511, Japan.

*Corresponding author: e-mail: Shisei Kubo email: kubo@nagasaki-u.ac.jp, Phone 81-95-819-7757, Fax:81-819-7757

Keywords: non-carious cervical lesion, morphology, progression, longitudinal study, swept-source optical coherence tomography 


\section{A pilot study to assess the morphology and progression of non-carious cervical lesions}

Abstract

Objective: This longitudinal pilot study aimed to morphologically and quantitatively investigate the progress of non-carious cervical lesions (NCCLs) by using swept-source optical coherence tomography (SS-OCT).

Methods: The samples examined comprised sets of NCCL epoxy resin replicas obtained from 10 lesions in 6 patients who attended annual dental visits over 4 or 5 years. SS-OCT images of the replicas were analyzed in terms of the maximum depth $\left(D_{\max }\right)$ and corresponding vertical width (VW) - using an image analyzer to estimate progression of the NCCLs over time.

Results: It was found that differences between wedge- and saucer-shaped lesions were morphologically distinguished well by the OCT images. There were significant differences in dimensions among $\mathrm{D}_{\max }$, VW and horizontal width (HW). HW was the largest and $\mathrm{D}_{\max }$ was the smallest. Although no significant differences in absolute values of annual progression rates were found among $\mathrm{D}_{\max }, \mathrm{VW}$ and $\mathrm{HW}$, the percentage increase in $\mathrm{D}_{\max }$ was significantly greater compared to VW and HW. The ratios of $\mathrm{D}_{\max }$ to corresponding VW ranged from 0.49 to 1.01 for the wedge-shaped lesions and from 0.13 to 0.44 for saucer-shaped lesions, respectively.

Conclusions: The dimensional analysis demonstrated notable progression with large variations. The wedge-shaped lesions appeared to show greater $D_{\max }$ values compared to the saucer-shaped lesions.

Clinical significance: With respect to the depth, the wedge-shaped lesions may progress at a greater rate compared to the saucer-shaped lesions. 


\section{Introduction}

Non-carious cervical lesions (NCCLs), which are characterized by loss of hard tissue at the cemento-enamel junction in the absence of caries, are commonly encountered in dental practice [1]. NCCLs are often restored due to hypersensitivity, poor esthetic appearance and prevention of further loss of tooth tissues [2]. However, Lyttle et al. [1] concluded that treatment for NCCLs may not be based on the correct diagnosis.

Generally, morphological features of NCCLs have been characterized as two distinct patterns, i.e. wedge-shaped lesions with sharp internal line angles and saucer-shaped lesions with more broad and rounded line angles [3]. For the wedge-shaped lesion, Aw et al. [4] introduced an angular scale of NCCLs, i.e. $<45^{\circ}, 45^{\circ}-90^{\circ}, 90^{\circ}-135^{\circ},>135^{\circ}$ as one of the parameters to characterize NCCL morphology. There is some suggestion that these morphological features of NCCLs are related to their etiology $[5,6]$. Wedge-shaped lesions could be caused by abrasive factors such as incorrect tooth-brushing habits or tooth loading causing high stresses due to bruxism, which is called abfraction, whereas saucer-shaped lesions are generally shallow and could be caused by acid erosion [7]. It is commonly accepted that initiation and progression of NCCLs has a multi-factorial etiology, but the relative contributions of the various etiological factors still remain unclear [4,8-12].

So far, most of the epidemiological surveys have been restricted to cross-sectional studies, where mostly visual inspection using scoring criteria of the Tooth Wear Index (TWI) was employed as a diagnostic tool [13]. Estafan et al. [10] further classified wedge-shaped lesions into 3 levels, mild (slight), moderate $(<1 \mathrm{~mm}$ deep) and severe $(\geq 1 \mathrm{~mm}$ deep). However, TWI is not suitable for quantitatively monitoring NCCL progression due to its large resolution. Very few studies have been published examining the longitudinal progression of NCCLs, where NCCL casts prepared by impression materials were obtained to record the 3-dimensional change over time [14]. This method may overcome, to some extent, the problem of subjective diagnosis of NCCLs by visual inspection. Another group employed 
micro-computed tomography to characterize NCCL morphology in-vitro and examined the abfraction theory using extracted teeth [3].

Optical coherence tomography (OCT) uses low coherence interferometry to determine the echo time delay and magnitude of backscattered light reflected from a biological structure [15]. This emerging diagnostic method can noninvasively obtain cross-sectional internal images of biological structures in real-time without use of x-ray irradiation. Swept-source OCT is the latest implementation of the technology, which has demonstrated superior sensitivity, higher scanning speed, and a higher signal-to-noise ratio over the conventional time-domain OCT [16]. For instance, studies on detection and quantification of de-/remineralization in enamel and dentin have been performed both in-vitro and in-vivo $[17,18]$. Other groups examined gap formation at the interface between the cavity floor and resin by OCT in-vitro [19]. Wada et al. [20] employed OCT to explore possible associations between NCCL size and other clinical observations such as occlusal wear, cracking, hypersensitivity and demineralization in-vivo.

A systematic review [11] revealed that there were several prospective studies that dealt with progression of NCCLs [14,21]. Recently, sets of NCCLs dental stone casts, which were annually prepared up to a maximum of 5 years from respective patients, were provided to our group to analyze NCCL progression using OCT. The purposes of this pilot study were to investigate the feasibility of OCT for NCCL morphological characterization and examine the progression over time by using the casts. The null hypotheses for this study were that OCT images do not clearly distinguish wedge- and saucer-shaped NCCLs, and the image analysis does not provide definitive data on the longitudinal changes in the dimensions of NCCLs. The other null hypothesis was that there is no association between morphology and progression rates of NCCLs.

\section{Materials and methods}




\subsection{Preparation of plaster impression casts of dentitions and its epoxy resin replicas}

A total of 26 subjects with NCCLs were recruited at Nagasaki University Hospital, Japan by one of the authors (SK) during the period between 2000 and 2005 to investigate the progression of NCCLs. This study was approved by the Ethics Committees of Nagasaki University School of Dentistry (No. 20). After careful removal of debris attached to tooth surfaces, silicone impressions (Exafine putty type, Injection type: GC Corp, Tokyo, Japan) of the NCCLs were taken and then poured up in improved dental stone (Fuji Rock: GC Corp). The stone casts were usually obtained every year for 4 or 5 years for each subject included in the study. These were transferred from Nagasaki University (NU) to Tokyo Medical and Dental University (TMDU) after collaboration between NU and TMDU was approved by the respective IRBs (No. 14070391 for NU and No. 959 for TMDU).

Ten sets of casts from 6 subjects, who fulfilled 4 or 5 years of observation and were randomly selected, were prepared for the current pilot study using OCT. Table 1 shows patients' ages and gender, the tooth code with NCCL, and year of data acquisition. The gingival margins of the casts covering the hidden portions of NCCLs were trimmed to allow OCT visualization of the areas that would have been covered by gingival tissue on the casts. In order to avoid damaging the original casts, a further impression using silicone impression material (Duplicone: Shofu Inc., Kyoto, Japan) was taken to duplicate the lesions without under-cut area. Replicas were prepared using transparent epoxy resin (EpoxiCure: Buehler, Lake Bluff, Illinois, USA).

\subsection{OCT image capturing of NCCL replicas}

This study used Swept-Source Optical Coherence Tomography (SS-OCT: Santec OCT-2000®, Santec Co., Komaki, Japan, Fig. 1a). The SS-OCT system incorporates a high-speed frequency swept external cavity laser, the wavelength ranges from $1260 \mathrm{~nm}$ to $1360 \mathrm{~nm}$ (centered at $1310 \mathrm{~nm}$ ) at a $20-\mathrm{kHz}$ sweep rate. Two-dimensional cross-sectional images were created from serial back-scatter (reflective) profiles along the surface. The axial 
and lateral resolutions of the system in air were 11 and $17 \mu \mathrm{m}$ respectively. The system acquired an output of the NCCL image data $(2000 \times 1019$ pixels $)$ in 0.3 sec including the processing time. The sensitivity of this SS-OCT and short-noise limited sensitivity were 106 $\mathrm{dB}$ and $119 \mathrm{~dB}$ respectively. The schematic presentation of SS-OCT laser optical circuit is available in the literature [20].

The epoxy resin replicas were fixed to a silicone holder placed on the stage (Fig. 1b) with the replica surface facing the scanning probe connected to the SS-OCT. The silicone holder was used to keep a line connecting the occlusal margin and the gingival margin at the center of the tooth in a mesiodistal direction parallel to both the surface and Y axis of the stage (Fig. 1b, c). The silicone holder was prepared for each set of NCCL epoxy resin replicas obtained from 10 lesions. In addition, the replica was placed so that the tooth axis was parallel to the scanning direction (Fig. 1c). The hand-held probe was set at a distance of $50 \mathrm{~mm}$ from the replica surface, with the scanning beam was oriented at 90 degrees to the surface (Fig. 1b). To enable the repeatability of the OCT scan for different replicas prepared for each observation time from the same subject, the replicas were placed at the same orientation as accurately as possible.

\subsection{Analysis of OCT image of NCCL replica}

A typical two-dimensional OCT image of a NCCL is shown in Figure 2. The white cross-section outline is of the NCCL surface which is apparent owing to strong light reflection at the lesion surface. The b-scan was performed from the mesial to distal ends of the lesion at $500 \mu \mathrm{m}$ intervals (Figure 1-c) and the NCCL was characterized in terms of the maximum depth $\left(\mathrm{D}_{\max }\right)$ and the corresponding vertical width (VW) as shown in Figure 2. A custom code in the image analysis software (ImageJ ver.1.47 n; Wayne Rasband, NIH, Bethesda, MD, USA) was used to handle the raw data of the OCT scan. The horizontal width (HW) was measured with a Vernier micrometer (CD-S15C, Mitutoyo Corp. Tokyo Japan) manually because of the large width of HW that could not be measured by the current OCT system. In 
this study, as the representative data for a single NCCL sample, the $\mathrm{D}_{\max }$ value and the corresponding VW value were employed. The progression rates of the lesion were determined by the difference in dimensions of 3 parameters between baseline and respective recalls. The morphological features of the 10 NCCLs were subjectively categorized by the first author referring to the traditional classification i.e., wedge-shaped lesions with sharp internal line angles, saucer-shaped lesions with broad and round line angles and mixed-shape lesions [3]. These analyses were performed for each observation year from the 10 lesions.

\subsection{Statistical analysis}

The Kruskal-Wallis test was used to compare the sizes, annual progression rates and the percentage increase between the 3 parameters by using SPSS statistical software (version 19.0, SPSS Inc., Chicago, Illinois, USA) at a significance level of 0.05 .

\section{Results}

Fig. 3 shows representative OCT images of the three shapes of NCCLs. Ten NCCLs observed in this study were categorized into the wedge-shaped lesion $(n=3)$, the saucer-shaped lesion $(n=6)$ and the mixed-shape lesion $(n=1)$. Fig. 4 shows a series of NCCL OCT images observed longitudinally for 4 years. In this case (L9), the lesion first appeared as a mixed-shaped lesion, then became more angular with the passage of time, and finally transformed into a wedge-shaped lesion.

Fig. 5, 6 and 7 exhibit longitudinal changes in D $\max$, VW and HW values during the $4-5$ years of evaluation respectively. It was shown that 7 out of the 10 lesions exhibited a decrease or regression in the values of $\mathrm{D}_{\max }, \mathrm{VW}$ and HW during the 4- or 5-year observation. These phenomena were unexpected, but all of the NCCLs showed an increase in the values at the final recall compared to the baseline.

The shapes, baseline and 4-year values, annual progression rates and percentage increase 
for the 10 NCCLs are summarized in Table 2. Despite a wide variation of values within each parameter, there were significant differences in dimensions among $\mathrm{D}_{\max }$, VW and HW. HW was the largest and $\mathrm{D}_{\max }$ was the smallest. Although statistical comparison between the shapes of NCCLs was not performed due to an insufficient sample size, the wedge-shaped lesions appeared to show greater $D_{\max }$ values compared to the saucer-shaped lesions. The ratios of $\mathrm{D}_{\max }$ to corresponding VW ranged from 0.49 to 1.01 for the wedge-shaped lesions and from 0.13 to 0.44 for the saucer-shaped lesions. Similarly, there seemed to be a significant difference in the ratios among the shapes.

By assuming the trends were linear during the follow-up period of 4 or 5 years, annual progression rates were calculated. No significant differences in annual progression rates were seen between the 3 parameters. In terms of the percentage increase, however, significant differences were found between $\mathrm{D}_{\max }$ and the others. There appeared to be two different trends in annual progression rates for $\mathrm{D}_{\max }$ as the three wedge-shaped lesions demonstrated higher annual progression rates than all saucer-shaped lesions. On the other hand, there seemed to be no significant differences between the shapes of NCCLs in terms of the percentage increase. With respect to both annual progression rates and the percentage increase for VW and HW, no apparent association was observed between their values and the shapes of NCCL.

\section{Discussion}

To our knowledge, there are a few studies reporting longitudinal observations of NCCL progression in the literature $[14,21,22]$. This is probably due to several difficulties such as lack of sensitive diagnostic tools to detect a small change over time in morphological parameters and the numerous efforts to prepare the NCCL casts in-vivo [14,22]. 3D scanning systems, the accuracy and the technology of which have been remarkably improved and/or progressed, may be useful to solve these problems. In the present study, however, a series of buccolingual outlines of each NCCL were captured using the SS-OCT. From these 
cross-sectional images, the depth and axial length were measured as accurately as possible using the ImageJ software. It has been demonstrated that the SS-OCT system is also clinically applicable and enables real-time capturing of NCCL images without taking impressions and manufacturing casts [20]. Therefore, a challenge to longitudinal studies on the effect of potential factors on the initiation and progression of NCCLs may be more easily conducted.

A systematic review revealed a wide variety of classifications and diagnostic criteria for the morphological features of NCCLs [11]. With respect to the angular characteristics of the NCCLs, an attempt to measure the internal line angle using three reference points in the vertical buccolingual section was made similar to a previous study [4]. One point was located at the deepest point on the lesion floor and the remaining 2 points were set at the occlusal and gingival margins of the NCCL. However, it was difficult to standardize the measurement of the internal line angle since the NCCLs did not always show a typical geometric triangular shape in the lesion. In addition, curved walls and rounded edges of lesions were often a characteristic. Further consideration is needed on how to objectively represent the angular characteristics. Thus NCCL categorization was performed subjectively on what has already been presented in the literature [3,4,23]. In this pilot study, 3, 6 and 1 NCCLs were classified into the wedge-, saucer- and mixed-shapes, respectively. The percentage distribution of wedge- and saucer-shaped lesions was also variable [3,4,9,23], which may be accounted for by age, population and subjective visual inspection criteria [11].

This study revealed difficulties in measuring the longitudinal data of $\mathrm{D}_{\max }$, VW and HW. All data should have exhibited a continuous increase in the values of $\mathrm{D}_{\max }$, VW and HW over time. However, 7 out of the 10 lesions exhibited a decrease between some recall intervals during the observation period as shown in Fig. 5 to 7. One possible reason for this fluctuation was difficulty in the accuracy to set the 3 measurement points. These points tended to be subjectively placed (Fig. 2) because all NCCLs did not always have sharp edges. Another reason is the possibility of a change in the baseline position of the tooth tissues due to further 
abrasive loss and thus losing the original reference point for measurements. Although fluctuations were observed over time in several lesions as mentioned above, the values of the 3 parameters at the final observation were greater than those at the baseline. In addition, changes in depths in the scans adjacent to the scan with $D_{\max }$ showed similar values to the $D_{\max }$ Therefore, we assumed that data obtained in this study were reliable.

With respect to the dimensions of NCCLs, HW was significantly larger than VW and Dmax. In addition, VW was significantly greater than $\mathrm{D}_{\max }$ as reported in previous studies $[4,9]$. This dimensional characteristic of NCCLs is probably due to the etiology of NCCLs and anatomical forms of teeth. Therefore, despite no significant difference in annual progression rates among the 3 parameters, the percentage increase for $D_{\max }$ was significantly greater compared to those for VW and HW. From a clinical practice point of view, $\mathrm{D}_{\max }$ seems to be the most crucial factor since it may have a great influence on decision making for treating NCCLs. In order to prevent further tooth tissue loss or tooth fracture, to relieve hypersensitivity and to recover esthetics, NCCLs tend to be restored as they become deeper. It has been reported that saucer-shaped lesions generally had a greater height (VW), a greater width (HW) and a smaller depth $\left(\mathrm{D}_{\max }\right)$ than wedge-shaped lesions [9]. However, in the present study, there seemed to be a significant difference only in depth between the shapes of NCCLs. Since the ratio of $\mathrm{D}_{\max }$ to corresponding VW of the wedge-shaped lesion was likely to be significantly greater than that of the saucer-shaped lesion, this ratio may be able to define the morphological characteristics of NCCLs. $D_{\max }$ values at 4 -year recall of the wedge-shaped lesions were greater than those of the saucer-shaped lesions. It has been speculated that the initiation and progression of NCCLs are related to many factors, such as tooth brushing habit, dietary acid ingestion and heavy occlusal stresses [3,4,9,12,24]. Although it seems to be very difficult to specify causal agents [11], mechanisms of wear or a combination of factors may be responsible for differences in the shapes and progression of NCCLs [12]. 
In this study, the mean annual progression rates were estimated using the values at the baseline and final observations by assuming that the progression was linear over time. The results indicated that the rates varied widely, suggesting a highly patient-dependent progression with several to ten times difference. Pintado et al. [14] monitored NCCL progression of a single adult aged 30 years at the start of the study over a 14-year time span using epoxy resin replicas. They calculated the mean depth by measuring the total tissue loss over the area of the respective NCCLs. The annual progression rates of mean depth between 8 and 14 years could be estimated to be $13 \mu \mathrm{m}$ for first premolar, $21 \mu \mathrm{m}$ for second premolar and $64 \mu \mathrm{m}$ for the first molar, respectively. These values are likely to be smaller compared to our results. This is probably because of the difference in the way of measuring the depth and the representation of the characteristics of NCCLs. In the present study, the maximum depth value $\left(D_{\max }\right)$ and the corresponding VW value were employed as the representative data for a single NCCL. Another possible reason is that our study included many older patients.

If the tooth loss continues with the calculated annual rate over a lifetime, most of the tooth tissue might be lost. However, clinical investigations revealed that more than 90 percent of the NCCLs had axial depths of less than $2 \mathrm{~mm}[4,13]$. In addition, about $75 \%$ of the NCCLs showed sclerosis and lack of sensitivity [4]. This might suggest that NCCL progression is a slow process. Another explanation is that the progression of the NCCLs may be arrested or slowed after reaching a plateau point. Annual progression rates and percentage increases may indicate that L1 and L3 were in the active stage, whereas L4 and L7 were in the inactive stage.

According to the results of this study, the null hypotheses that OCT images do not clearly distinguish wedge- and saucer-shaped NCCLs, and the image analysis does not provide definitive data on longitudinal changes in dimensions of NCCLs were rejected. The other hypothesis that there is no association between morphology and progression rates of NCCLs might be partly accepted. 
Laser scanning microscopy is also a useful means to analyze the morphological and geometric changes of the specimens in the laboratory. It has a similar accuracy as SS-OCT. The 3D image data obtained from laser scanning microscopy may be more effectively and quantitatively analyzed by using the current software. Further study is required to increase the sample size using the remaining stone casts of the NCCLs. The relationship between NCCLs and their potential causative factors that have already been collected from the subjects by a questionnaire will be also investigated. Moreover, a longitudinal clinical study using SS-OCT is planned to be conducted to explicate the initiation and the progression of NCCLs as well as to confirm the findings of the present study.

\section{Conclusion}

Although there were several limitations in this pilot study, the SS-OCT system could distinguish morphological differences between wedge-shaped and saucer-shaped lesions. The dimensional analysis demonstrated notable progression with large variations. Although no significant differences in absolute values of annual progression rates between depth $\left(D_{\max }\right)$, vertical width (VW) and horizontal width (HW), the percentage increase in $\mathrm{D}_{\max }$ was significantly greater compared to VW and HW. The ratios of $\mathrm{D}_{\max }$ to corresponding VW ranged from 0.49 to 1.01 for the wedge-shaped lesions and from 0.13 to 0.44 for the saucer-shaped lesions, respectively. With respect to the depth, the wedge-shaped lesions tended to progress at a greater rate compared to the saucer-shaped lesions. 


\section{References}

[1] H.A. Lyttle, N. Sidhu, B. Smyth, A study of the classification and treatment of noncarious cervical lesions by general practitioners, J. Prosthet. Dent. 79 (1998) 342-346.

[2] Morigami M, Sugizaki J, Uno S, Yamada T, A clinical assessment of resin composite restorations, Adhes Dent. 38 (2007) 177-181

[3] B. Hur, H.C. Kim, J.K. Park, A. Versluis, Characteristics of non-carious cervical lesions an ex vivo study using micro computed tomography, J. Oral Reahabil. 38 (2011) 469-474.

[4] T.C. Aw, X. Lepe, G.H. Johnson, L. Mancl, Characteristics of noncarious cervical lesions: a clinical investigation, J. Am. Dent. Assoc. 133, (2002) 725-733.

[5] R.F. Sognnaes, R.B. Wolcott, F.A. Xhonga, Erosion-like patterns occurring in association with other dental conditions, J. Am. Dent. Assoc. 84 (1972) 571-576.

[6] W.C. Lee, W.S. Eakle, Possible role of tensile stress in the etiology of cervical erosive lesions of teeth, J. Prosthet. Dent. 52 (1984) 374-380.

[7] L.C. Levitch, J.D. Bader, D.A. Shugars, H.O. Heymann, Non-carious cervical lesions, J. Dent. 22 (1994) 195-207.

[8] J.O. Grippo, M .Simring, T.A. Coleman, Abfraction, abrasion, biocorrosion, and the enigma of noncarious cervical lesions: A 20-Year Perspective, J. Esthet. Restor. Dent. 24 (2012) 10-23.

[9] B.T. Piotrowski, W.B. Gillette, EB Hancock, Examining the prevalence and characteristics of abfraction like cervical lesions in a population of U.S. veterans, J. Am. Dent. Assoc. 132 (2001) 1694-1701.

[10] A. Estafan, P.C. Furnari, G. Goldstein, E.L. Hittelman, In vivo correlation of noncarious cervical lesions and occlusal wear, J. Prosthet. Dent. 93 (2005) 221-226.

[11] P. Senna, A.D.B. Cury, C. Rösing, Non-carious cervical lesions and occlusion: a systematic review of clinical studies, J. Oral Rehabil. 39 (2012) 450-462.

[12] C. Walter, E. Kress, H. Götz, K. Taylor, I. Willershausen, A. Zampelis, The anatomy of 
non-carious cervical lesions, Clin. Oral Invest. 18 (2014) 139-146.

[13] J. Borcic, I. Anic, M.M. Urek, S. Ferreri, The prevalence of non-carious cervical lesions in permanent dentition, J. Oral Rehabil. 31 (2004) 117-123.

[14] M.R. Pintado, R. Delong, C.C. Ko, R.L. Sakaguchi, W.H. Douglas, Correlation of noncarious cervical lesion size and occlusal wear in a single adult over a 14-year time span, J. Prosthet. Dent. 84 (2000) 436-443.

[15] D. Huang, E.A. Swanson, C.P. Lin, J.S. Schuman, W.G. Stinson, W, Chang, et al., Optical coherence tomography, Science. 254 (1991) 1178-1181.

[16] M. Choma, M. Sarunic, C. Yang, J. Izatt, Sensitivity advantage of swept source and Fourier domain optical coherence tomography, Opt. Express. 11 (2003) 2183-2189.

[17] Y. Natsume, S. Nakashima, A .Sadr, Y. Shimada, J. Tagami, Y. Sumi, Estimation of lesion progress in artificial root caries by swept source optical coherence tomography in comparison to transverse microradiography, J. Biomed. Opt. 16 (2011) 071408.

[18] Y. Shimada, H. Nakagawa, A. Sadr, I. Wada, M. Nakajima, T. Nikaido, et al., Noninvasive cross-sectional imaging of proximal caries using swept-source optical coherence tomography (SS-OCT) in vivo, J. Biophotonics 7 (2014) 506-513.

[19] T.A. Bakhsh, A. Sadr, Y. Shimada, J. Tagami, Y. Sumi, Non-invasive quantification of resin-dentin interfacial gaps using optical coherence tomography: validation against confocal microscopy, Dent. Mater. 27 (2011) 915-925.

[20] I. Wada, Y. Shimada, M. Ikeda, A. Sadr, S. Nakashima, J. Tagami, et al., Clinical assessment of non carious cervical lesion using swept-source optical coherence tomography, J. Biophotonics 8 (2015) 846-854.

[21] A. Lussi, M. Schaffner, Progression of and risk factors for dental erosion and wedge-shaped defects over a 6-year period, Caries Res. 34 (2000) 182-187.

[22] I.D. Wood, A.S.A. Kassir, P.A. Brunton, Effect of lateral excursive movement on the progression of abfraction lesions, Oper. Dent. 34 (2009) 273-279. 
[23] N. Miller, J. Penaud, P. Ambrosini, C. Bisson-Boutelliez, S. Briançon, Analysis of etiologic factors and periodontal conditions involved with 309 abfractions, J. Clin. Periodontol. 30 (2003) 828-832.

[24] O. Bernhardt, D. Gesch, C. Schwahn, F. Mack, G. Meyer, U. John et al., Epidemiological evaluation of the multifactorial aetiology of abfractions, J. Oral Rehabil. 33 (2006) 17-25. 
Table 1 Year of data acquisition from the study lesions

\begin{tabular}{ccccccccccc} 
Lesion & Subject & Tooth code & Age & Gender & 2000 & 2001 & 2002 & 2003 & 2004 & 2005 \\
\hline L1 & S1 & 45 & 38 & F & $\bigcirc$ & $\bigcirc$ & $\bigcirc$ & $\times$ & $\bigcirc$ & $\times$ \\
L2 & S2 & 14 & 52 & F & $\bigcirc$ & $\times$ & $\bigcirc$ & $\times$ & $\bigcirc$ & $\times$ \\
L3 & S2 & 25 & 52 & F & $\bigcirc$ & $\bigcirc$ & $\bigcirc$ & $\times$ & $\bigcirc$ & $\times$ \\
L4 & S3 & 15 & 48 & M & $\bigcirc$ & $\times$ & $\bigcirc$ & $\bigcirc$ & $\bigcirc$ & $\bigcirc$ \\
L5 & S4 & 14 & 57 & F & $\times$ & $\bigcirc$ & $\bigcirc$ & $\bigcirc$ & $\bigcirc$ & $\bigcirc$ \\
L6 & S4 & 44 & 57 & F & $\times$ & $\bigcirc$ & $\bigcirc$ & $\bigcirc$ & $\bigcirc$ & $\bigcirc$ \\
L7 & S5 & 24 & 68 & F & $\bigcirc$ & $\bigcirc$ & $\bigcirc$ & $\times$ & $\times$ & $\bigcirc$ \\
L8 & S6 & 13 & 61 & F & $\times$ & $\bigcirc$ & $\times$ & $\times$ & $\bigcirc$ & $\bigcirc$ \\
L9 & S6 & 14 & 61 & F & $\times$ & $\bigcirc$ & $\bigcirc$ & $\times$ & $\bigcirc$ & $\bigcirc$ \\
L10 & S6 & 15 & 61 & F & $\times$ & $\bigcirc$ & $\times$ & $\times$ & $\bigcirc$ & $\bigcirc$ \\
\hline
\end{tabular}

$\circ$ : presence, $\times$ : absence 
Table 2 Progression of NCCLs $(\mu \mathrm{m})$

\begin{tabular}{|c|c|c|c|c|c|c|c|c|c|c|c|c|c|c|}
\hline \multirow[b]{2}{*}{ Lesion } & \multirow{2}{*}{$\begin{array}{l}\text { Tooth } \\
\text { Code }\end{array}$} & \multirow[b]{2}{*}{ Shape } & \multicolumn{4}{|c|}{$\mathrm{D}_{\max }$} & \multicolumn{4}{|c|}{ VW } & \multicolumn{4}{|c|}{ HW } \\
\hline & & & Baseline & $4 y$ & $\begin{array}{c}\text { Annual } \\
\text { rate }\end{array}$ & $\begin{array}{l}\% \\
\text { inc. }\end{array}$ & Baseline & $4 y$ & $\begin{array}{c}\text { Annual } \\
\text { rate }\end{array}$ & $\begin{array}{l}\% \\
\text { inc. }\end{array}$ & Baseline & $4 y$ & $\begin{array}{c}\text { Annual } \\
\text { rate }\end{array}$ & $\begin{array}{l}\% \\
\text { inc. }\end{array}$ \\
\hline L1 & 45 & Wedge & 539 & 1489 & 238 & 176 & 2290 & 3017 & 182 & 32 & 4095 & 5080 & 246 & 24 \\
\hline L2 & 14 & Saucer & 382 & 591 & 52 & 55 & 3039 & 3930 & 223 & 29 & 3320 & 3870 & 138 & 17 \\
\hline L3 & 25 & Saucer & 344 & 546 & 50 & 59 & 1542 & 2635 & 273 & 71 & 3480 & 3820 & 85 & 10 \\
\hline L4 & 15 & Saucer & 322 & 366 & 11 & 14 & 2642 & 2807 & 41 & 6 & 4250 & 4630 & 95 & 9 \\
\hline L5 & 14 & Saucer & 449 & 584 & 34 & 30 & 1445 & 1722 & 69 & 19 & 2970 & 3400 & 108 & 14 \\
\hline L6 & 44 & Saucer & 419 & 613 & 49 & 46 & 2433 & 2957 & 131 & 22 & 3760 & 4130 & 93 & 10 \\
\hline L7 & 24 & Saucer & 1115 & 1219 & 26 & 9 & 2465 & 2754 & 72 & 12 & 4260 & 4350 & 23 & 2 \\
\hline L8 & 13 & Mixed & 890 & 1182 & 73 & 33 & 1781 & 1901 & 30 & 7 & 6130 & 6840 & 178 & 12 \\
\hline L9 & 14 & Wedge & 1399 & 1968 & 142 & 41 & 1826 & 1939 & 28 & 6 & 4340 & 4930 & 148 & 14 \\
\hline L10 & 15 & Wedge & 1152 & 1564 & 103 & 36 & 1572 & 1811 & 60 & 15 & 4150 & 4280 & 33 & 3 \\
\hline
\end{tabular}

$\mathrm{D}_{\max }$ : maximum depth, VW: vertical width corresponded to $\mathrm{D}_{\max }, \mathrm{HW}$ : horizontal width, inc.: increase 
a)

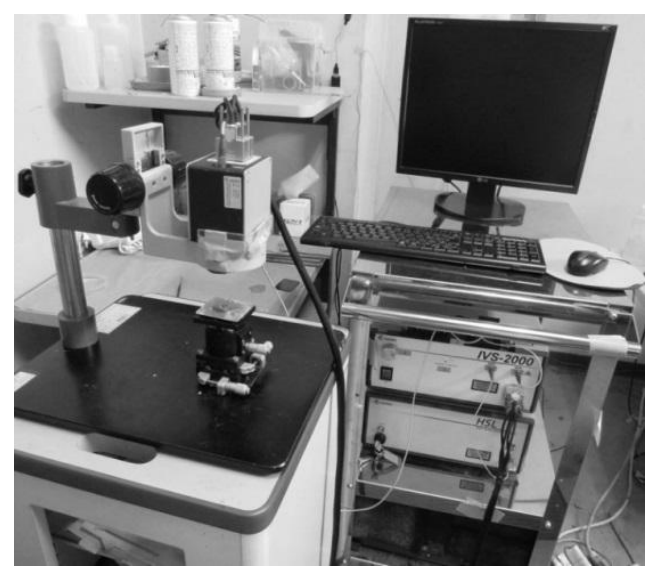

b)

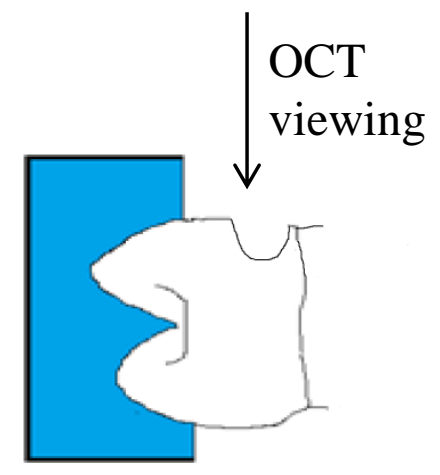

c)

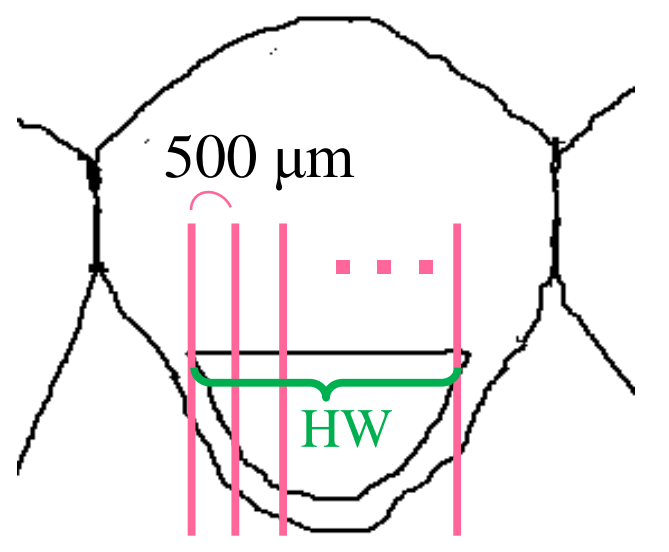

Fig. 1 


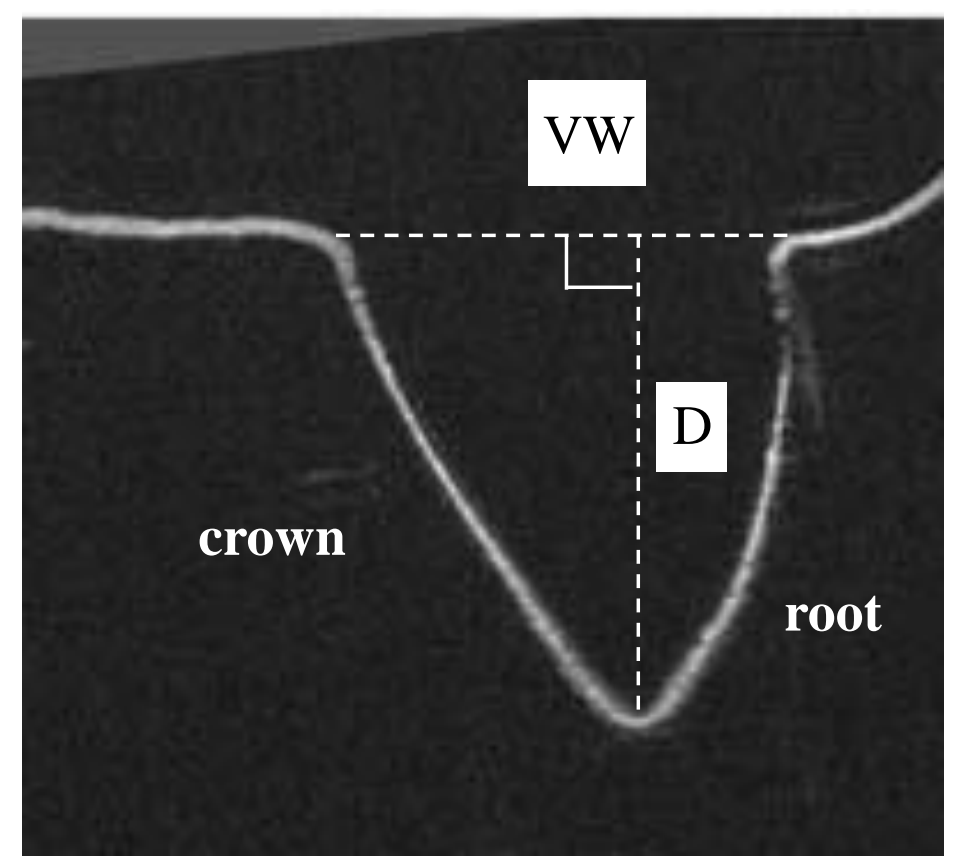

Fig. 2 

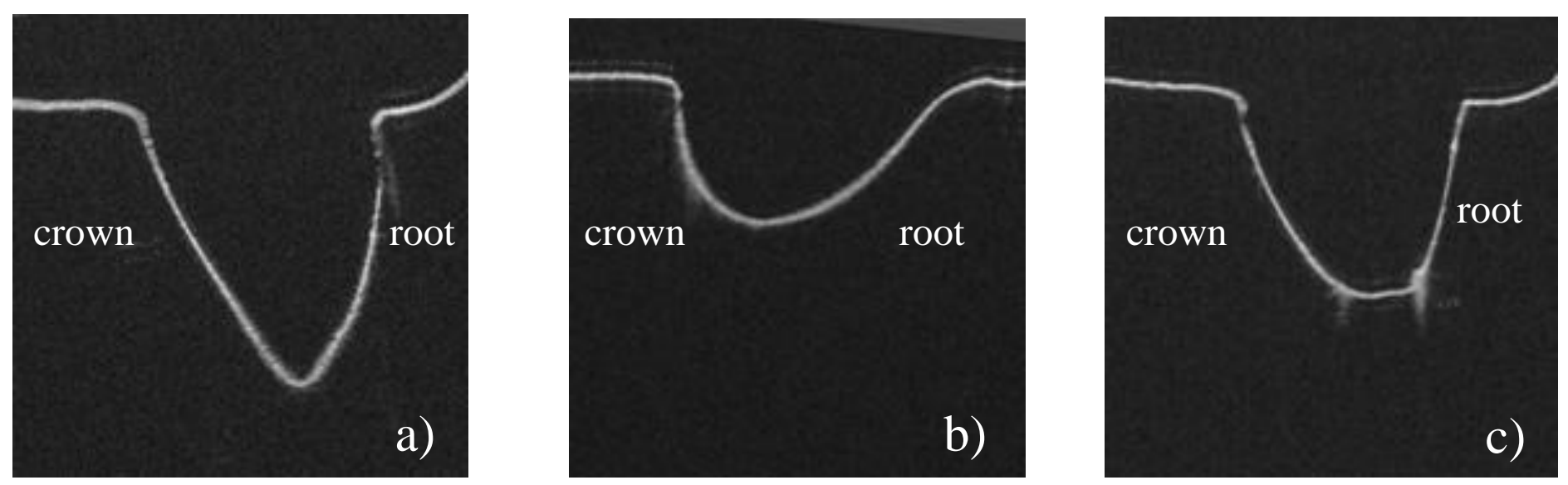

Fig. 3 


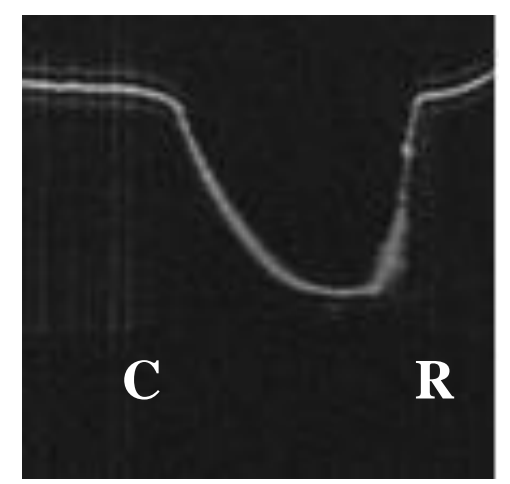

Baseline

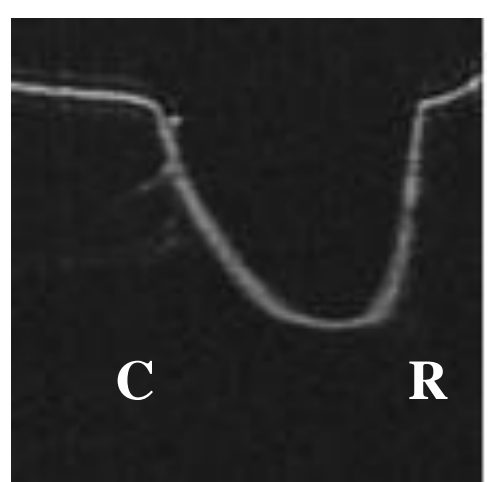

1 year

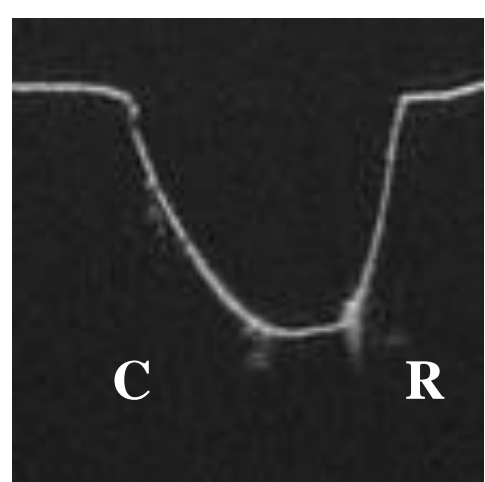

3 years

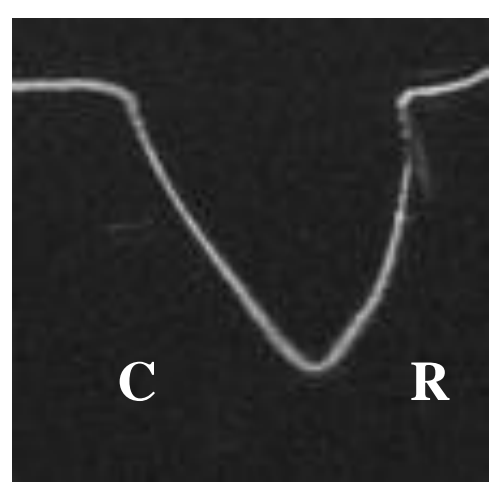

4 years

Fig. 4 


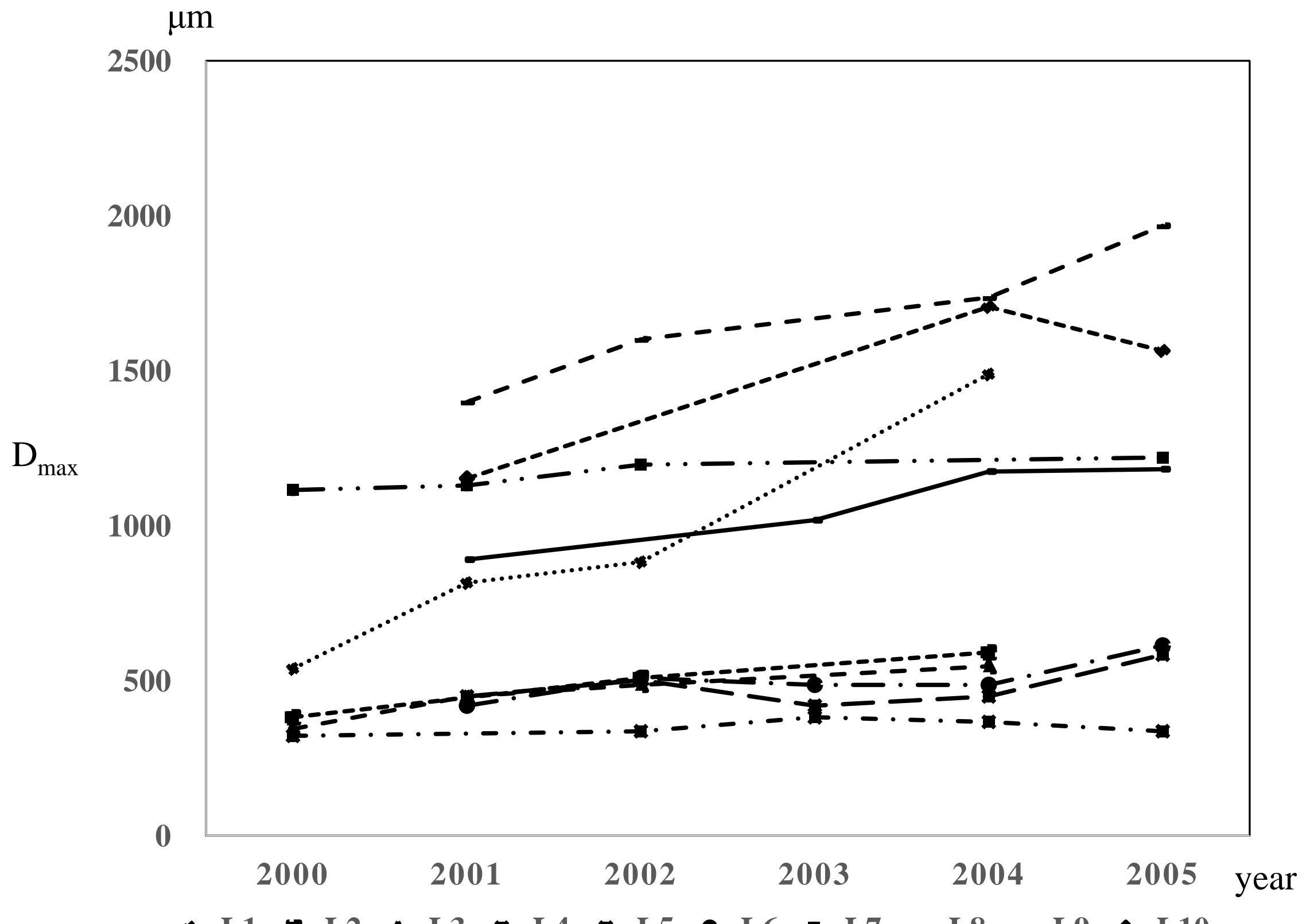




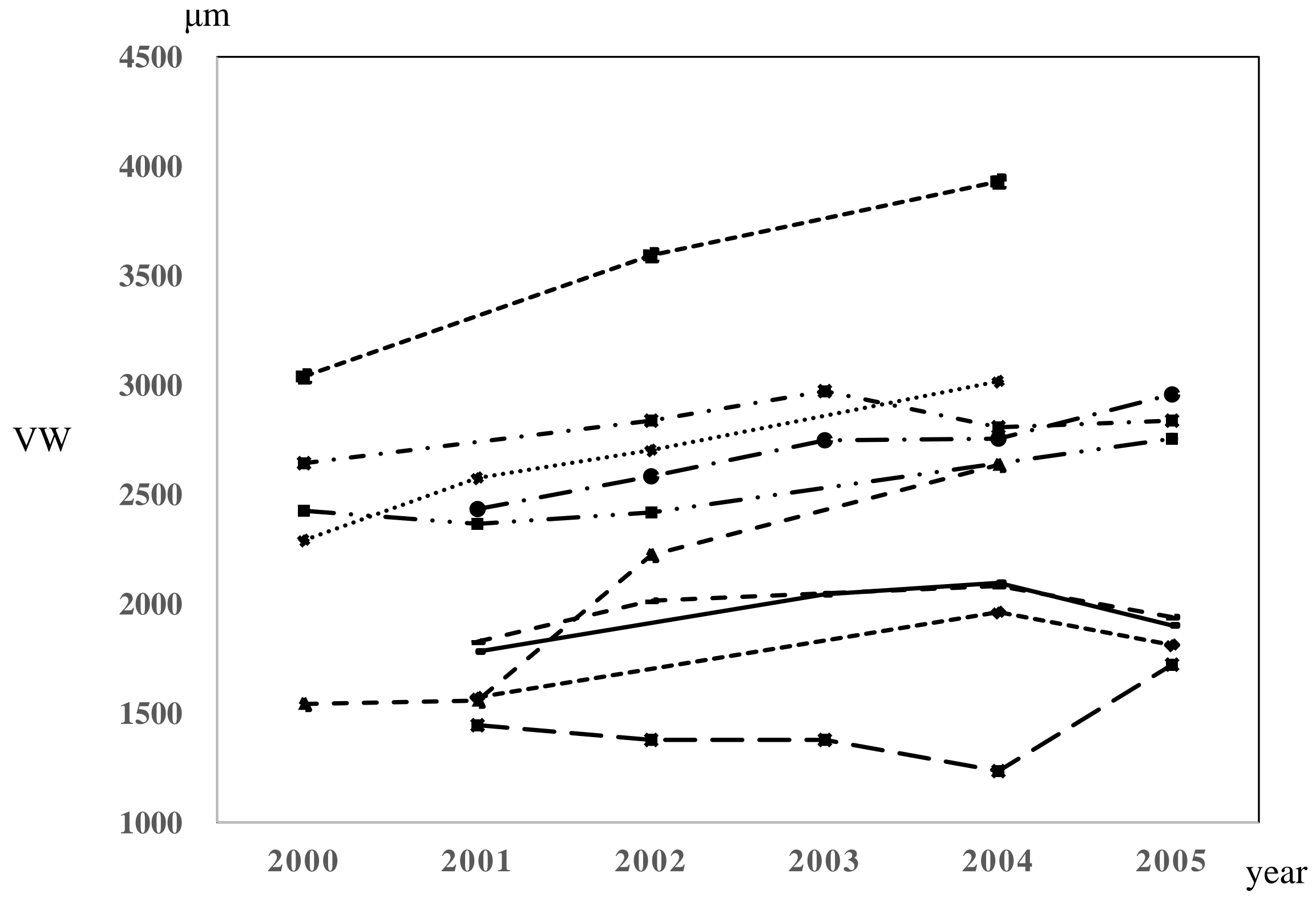

..*.. L1 - 


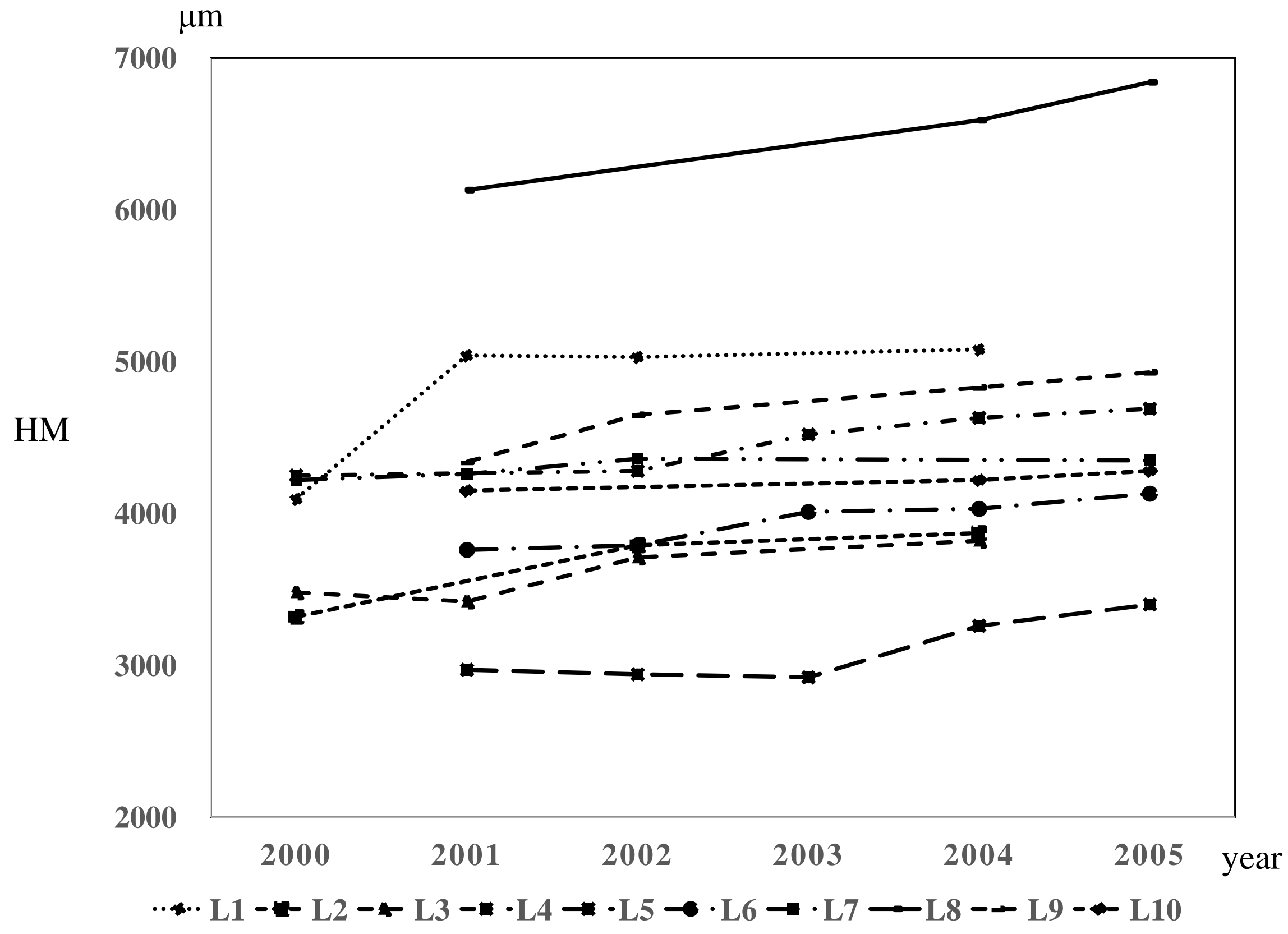

Fig. 7 


\section{Captions}

Fig. 1 Photograph and schemas of the SS-OCT system and image capturing

a): Overview of SS-OCT, b): The epoxy resin replica of the NCCL fixed to a silicone holder, c): OCT image capturing (b-scan) at $500 \mu \mathrm{m}$ intervals SS-OCT: swept-source optical coherence tomography; NCCL: the non-carious cervical lesion; HW: horizontal width of the NCCL.

Fig. 2 Analysis of OCT image in terms of depth (D) and vertical width (VW)

Fig. 3 Representative OCT images of the three shapes of NCCLs

a): wedge-shaped, b): saucer-shaped, c) : mixed-shaped

Fig. 4 A series of NCCL OCT images observed longitudinally for 4 years

C: crown site; R: root site. This lesion first appeared as a mixed-shaped lesion, then became more angular with the passage of time, and finally transformed into a wedge-shaped lesion.

Fig. 5 Progression of $D_{\max }$ for each lesion

$\mathrm{D}_{\max }$ : the maximum depth of the lesion

Fig. 6 Progression of VW for each lesion

VW: vertical width corresponded to $\mathrm{D}_{\max }$

Fig. 7 Progression of HW for each lesion

HW: horizontal width of the lesion 